\title{
Increase of interstitial glycerol reflects the degree of ischaemic brain damage: a PET and microdialysis study in a middle cerebral artery occlusion-reperfusion primate model
}

\author{
P Frykholm, L Hillered, B Långström, L Persson, J Valtysson, Y Watanabe, P Enblad
}

Department of

Neuroscience, Section of Neurosurgery, Uppsala University Hospital, S-751 85 Uppsala, Sweden P Frykholm

L Hillered

L Persson

P Enblad

Department of Medical Science, Section of Clinical Chemistry

L Hillered

Departments of Surgical Science and Anaesthesiology

J Valtysson

Uppsala University PET Centre Uppsala, Sweden

B Långström

Department of Physiology, Osaka City University Graduate School of Medicine and Department of

Neuroscience, Osaka Bioscience Institute, Osaka, Japan

Y Watanabe

Correspondence to: Dr P Enblad

Per.Enblad@neurokir.uu.se

Received 4 December 2000 and in revised form 19 April 2001

Accepted 4 June 2001

\begin{abstract}
Objective-To evaluate interstitial glycerol as a marker of ischaemia by studying the changes in glycerol in direct relation to changes in regional cerebral metabolic rate of oxygen $\left(\mathrm{CMRO}_{2}\right)$, the lactatel pyruvate ratio (LP ratio), and glutamate. Methods-Transorbital 2 hour middle cerebral artery occlusion (MCAO) was performed in eight monkeys, which were studied with continuous microdialysis for 24 hours. Interstitial fluids were collected by microdialysis and analysed for glycerol, lactate, pyruvate, and glutamate with an enzymatic assay and high performance liquid chromatography. Sequential PET studies of cerebral blood flow (CBF), $\mathrm{CMRO}_{2}$, oxygen extraction ratio (OER), and cerebral blood volume (CBV) were performed. The microdialysis probe regions were classified as severe ischaemia or penumbra, depending on whether the mean $\mathrm{CMRO}_{2}$ side to side ratio was below or above $60 \%$, respectively.

Results-A nine-fold, sustained increase in glycerol was registered after MCAO in severe ischaemia regions. In penumbra regions, the increase in glycerol was five-fold, but the glycerol concentration returned to baseline within 8 hours of clip removal. The difference between severe ischaemia and penumbra glycerol values was statistically significant. As expected from previous studies, the interstitial LP ratio and glutamate increased markedly in severe ischaemia, with a less pronounced change in penumbra regions. There was a time lag between the biochemical changes in severe ischaemia regions, with the LP ratio preceding glutamate, followed by glycerol.
\end{abstract}

Conclusions-A marked, sustained increase in interstitial glycerol is indicative of severe ischaemia in this stroke model. A

Table 1 Summary of the monitored physiological variables in the eight monkeys

\begin{tabular}{llllllll}
\hline & $B$ & $M C A O$ & $R 1$ & $R 2$ & $R 4$ & $R 8$ & $F$ \\
\hline $\mathrm{pCO}_{2}(\mathrm{kP})$ & $5.3(1.1)$ & $5.0(0.7)$ & $5.0(0.5)$ & $5.3(0.6)$ & $5.1(0.6)$ & $5.0(0.5)$ & $5.0(0.8)$ \\
$\mathrm{pO}_{2}(\mathrm{kP})$ & $18(6.8)$ & $18(6.7)$ & $19(8.3)$ & $18(7.2)$ & $18(6.0)$ & $20(7.0)$ & $18(5.8)$ \\
$\mathrm{Hb}(\mathrm{g} / \mathrm{l})$ & $112(23)$ & $103(24)$ & $95(21)$ & $98(21)$ & $95(20)$ & $105(5.8)$ & $86(21)$ \\
$\mathrm{MAP}(\mathrm{mm} \mathrm{Hg})$ & $108(17)$ & $100(18)$ & $104(21)$ & $96(14)$ & $88(14)$ & $84(11)$ & $96(29)$ \\
$\mathrm{Temp} .\left({ }^{\circ} \mathrm{C}\right)$ & $35.9(1.0)$ & $36.6(1.2)$ & $37.0(1.0)$ & $37.2(0.8)$ & $37.4(0.7)$ & $36.8(0.6)$ & $36.9(0.4)$ \\
$\mathrm{ICP}(\mathrm{mm} \mathrm{Hg})$ & $9(4)$ & $9(6)$ & $9(7)$ & $9(7)$ & $15(10)$ & $22(15)$ & $25(24)$ \\
\hline
\end{tabular}

Values are means (SD). B=baseline PET; $M C A O=$ middle cerebral artery occlusion PET; R1 through R8=1, 2, 4, and 8 hours of reperfusion, respectively; $\mathrm{F}=$ final examination. transient, diminutive increase in interstitial glycerol may reflect a penumbra situation. Interstitial glycerol in combination with other biochemical markers such as the LP ratio and glutamate may be useful for clinical monitoring of the ischaemic brain, reflecting a sequence of secondary pathophysiological events.

(F Neurol Neurosurg Psychiatry 2001;71:455-461) microdialysis

Cerebral ischaemia leads to the degradation of membrane phospholipids. ${ }^{12}$ This may lead to disturbed cell membrane function and cell death. $^{34}$ Glycerol is an end product of phospholipid breakdown in brain tissue and has been shown to be a promising marker of cell damage in traumatic brain injury in rats, ${ }^{5}$ and in patients with subarachnoid haemorrhage. ${ }^{6}$ However, the relation between cerebral ischaemia and changes in interstitial glycerol needs further illumination. We have studied the changes in interstitial energy related metabolites and excitatory amino acids as markers of ischaemia, simultaneously with regional PET measurements of cerebral blood flow (CBF), cerebral metabolic rate of oxygen $\left(\mathrm{CMRO}_{2}\right)$, and oxygen extraction ratio (OER) both in patients and in a recently developed middle cerebral artery occlusion (MCAO)-reperfusion experimental model in primates. ${ }^{7-9}$ The aim of the present study was to further evaluate interstitial glycerol as a marker of ischaemia, focusing on the changes in glycerol in direct relation to changes in regional $\mathrm{CMRO}_{2}$, the lactate/ pyruvate ratio (LP ratio), and glutamate.

Materials and methods

THE MCA OCCLUSION: REPERFUSION MODEL

The study included the same series of monkeys previously reported, eight adult Macaca Mulatta. ${ }^{9}$ The study protocol was approved by the
Keywords: interstitial glycerol; ischaemic brain damage; 


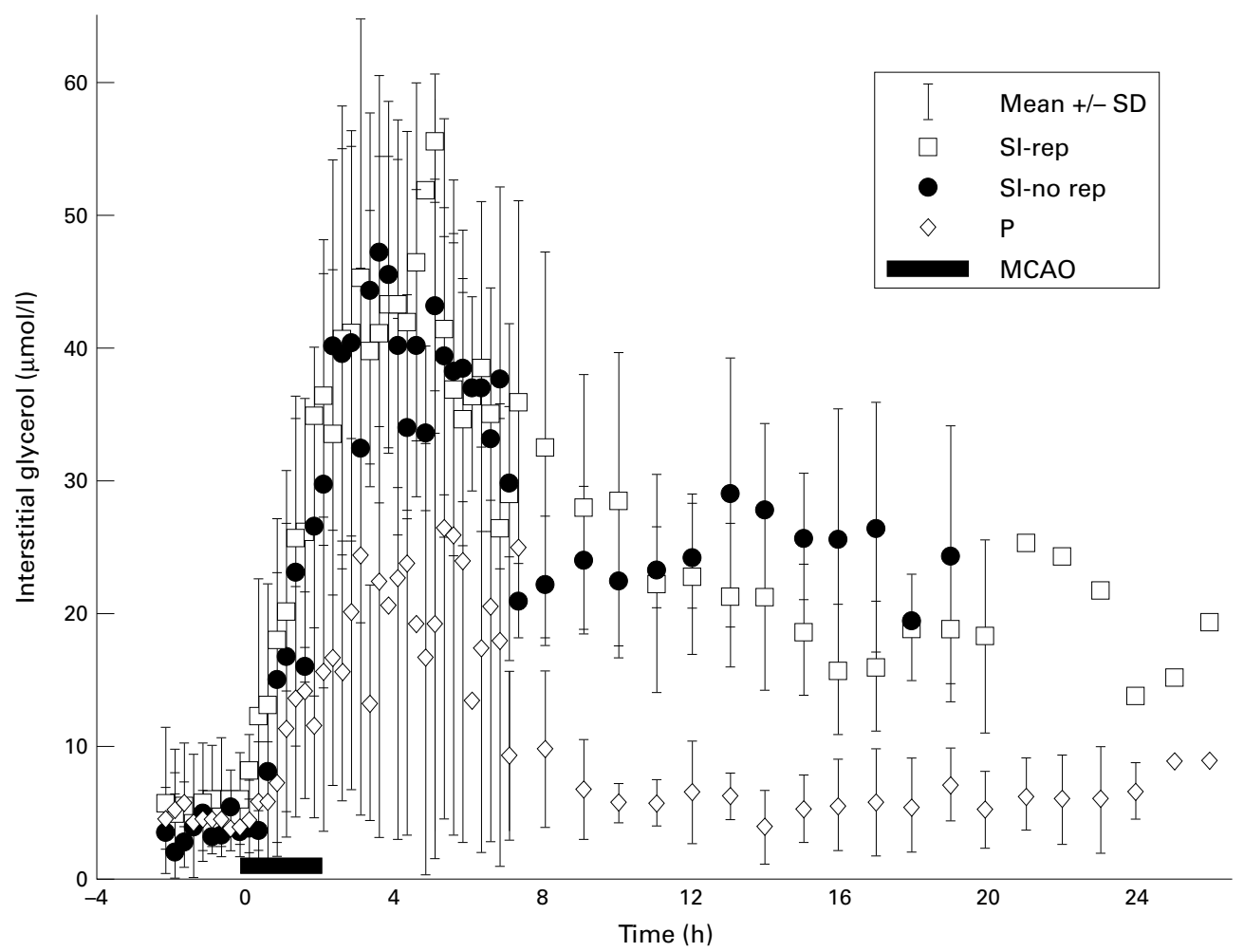

Figure 1 Mean glycerol values (SD) in severe ischaemia probe regions with reperfusion (SI-rep), severe ischaemia with no significant reperfusion (SI-no rep), and penumbra (P) probe regions.

local ethics committee for animal research (permissions c 38/96 and c 37/97). As described in detail in Frykholm et al, ${ }^{9}$ anaesthesia was maintained with a continuous infusion of midazolam $(0.1 \mathrm{mg} / \mathrm{kg} / \mathrm{h})$ and morphine $(0.1$ $\mathrm{mg} / \mathrm{kg} / \mathrm{h})$. Atracurium $(0.5 \mathrm{mg} / \mathrm{kg} / \mathrm{h})$ was used for muscle relaxation. A femoral artery was catheterised for blood pressure monitoring and blood sampling. Arterial blood pressure, electrocardiography, pulse oximetry, end tidal $\mathrm{CO}_{2}$, intracranial pressure, and rectal temperature were continuously monitored. A controlled heating mattress was used to maintain normothermia. The monitored physiological variables are shown in table 1.

Two microdialysis probes were inserted stereotaxically into the right hemisphere, within the deep and superficial regions of the MCA territory. ${ }^{8}$ After a baseline PET session, a microsurgical transorbital approach was used for MCAO. ${ }^{10}{ }^{11} \mathrm{~A}$ second PET was performed during MCAO. After 2 hours of MCAO the clip was removed and sequential PET measurements continued during reperfusion.

Table 2 Time profiles (median (range)) of the microdialysis parameters in all probes with severe ischaemia

\begin{tabular}{lll}
\hline Parameter & Response time* & Time to first peakt \\
\hline Severe ischaemia: & & \\
$\quad$ LP ratio & $15(15-30)$ & $45(15-120)$ \\
Glutamate & $30(15-60)$ & $75(45-120)$ \\
Glycerol & $37.5(15-60)$ & $157.5(90-210)$ \\
\hline
\end{tabular}

$\star$ Time (minutes) after MCAO for doubling of the mean baseline value.

†Time (in minutes) after MCAO when the MD parameter reached its first peak.
MICRODIALYSIS

Microdialysis monitoring was started immediately after probe insertion, and continued until the final PET session was completed. The two microdialysis probes (CMA/10, CMA/ Microdialysis, Stockholm, Sweden) had a membrane length of $4 \mathrm{~mm}$ and shaft lengths of $50 \mathrm{~mm}$ and $20 \mathrm{~mm}$. Artificial CSF (containing $148 \mathrm{mmol} / 1 \mathrm{Na}^{+}, 1.2 \mathrm{mmol} / 1 \mathrm{Ca}^{2+}, 0.9 \mathrm{mmol} / 1$ $\mathrm{Mg}^{2+}, 2.7 \mathrm{mmol} / 1 \mathrm{~K}^{+}$, and $155 \mathrm{mmol} / \mathrm{C} \mathrm{Cl}^{-}$,) was delivered as perfusion medium by a microinjection pump (CMA/100) at a rate of $2 \mu \mathrm{l} /$ minute. Samples were collected at 15 minute intervals until 4 hours after reperfusion, and thereafter every hour. Dead space from membrane to collecting vial was $13.6 \mu \mathrm{l}$ for the deep and 7.6 $\mu \mathrm{l}$ for the superficial probe respectively. Lactate, pyruvate, and glutamate were analysed by high performance liquid chromatography. ${ }^{12}{ }^{13}$ Glycerol was measured by enzymatic fluorometric assay on a CMA/600 analyser (CMA/ Microdialysis; see also Foster $e t a l^{14}$ ). This assay is a relatively new bedside method which, in our hands, has a total imprecision of $6.0 \%$ (coefficient of variation), and a within run imprecision of $3 \%$ at the $15 \mu \mathrm{mol} / 1$ concentration. ${ }^{6}$ Microdialysis data were presented as the dialysate concentrations without correction for in vivo probe recovery, because no method for repeated determination of in vivo probe recovery was available. ${ }^{615}{ }^{16}$ For the figures, it was not considered necessary to correct the time scale for dead space in the probe and outlet tubing, corresponding to a time lag of 6.8 and 3.8 minutes for the deep and superficial probes, respectively. 
PET

The PET investigations were performed on a GEMS 2048-15B scanner (General Electric Medical Systems, Milwaukee, WI) ${ }^{17}$ as described in detail earlier. ${ }^{9}$ A computerised reorientation procedure was used to accurately align the consecutive PET studies to enable exact intraindividual comparisons. ${ }^{18}$ Each PET scanning procedure consisted of three examinations with different tracers: Carbon $\left[{ }^{15} \mathrm{O}\right]$ monoxide, Carbon $\left[{ }^{15} \mathrm{O}\right]$ dioxide, and $\left[{ }^{15} \mathrm{O}\right]$ oxygen. This allowed calculations of cerebral blood volume (CBV), cerebral blood flow (CBF), $\mathrm{CMRO}_{2}$, and oxygen extraction rate (OER), using the
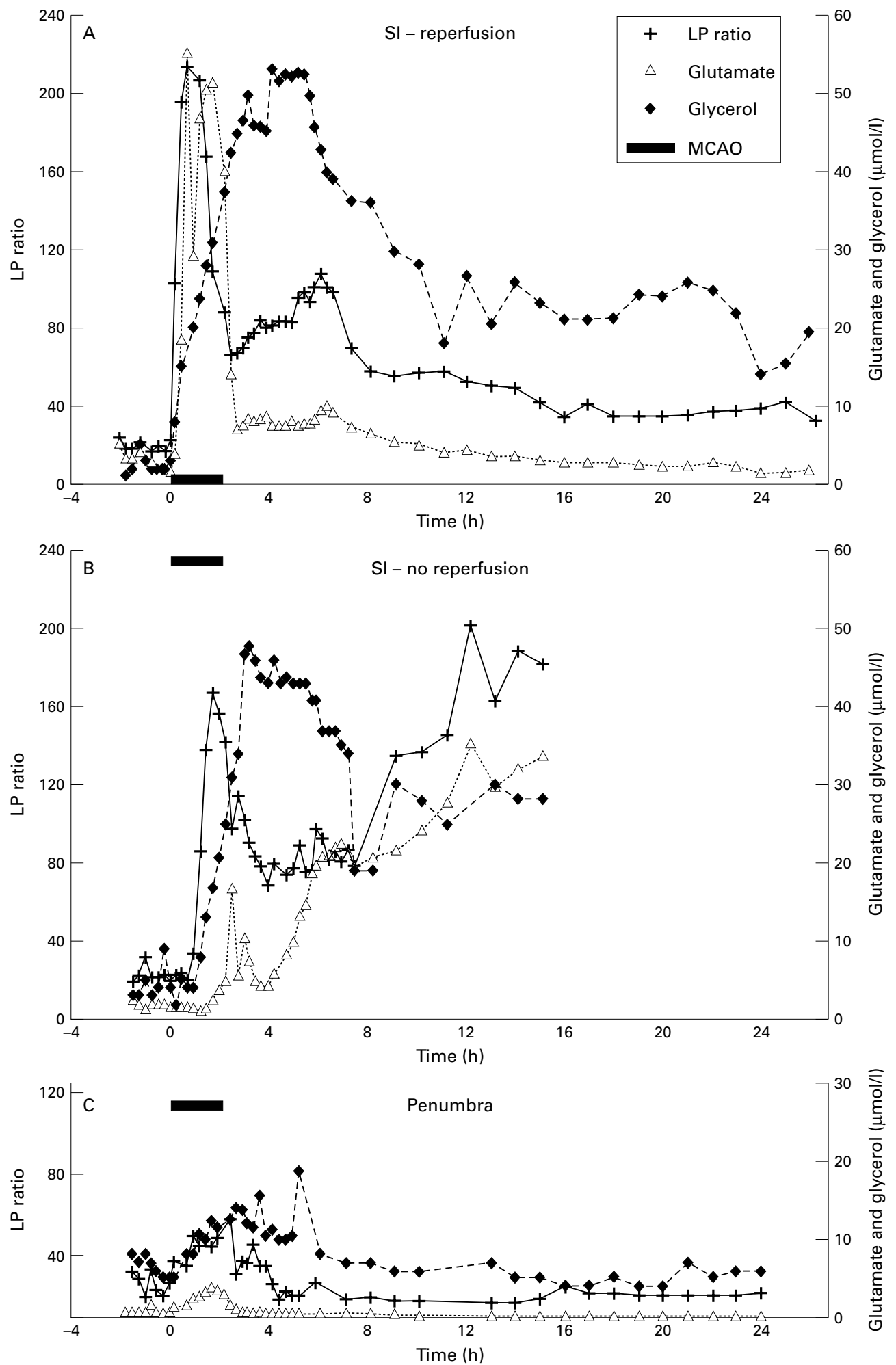

Figure 2 Microdialysis time profiles in probe regions located in three different types of ischaemic tissue-severe ischaemia $(S I)$ with reperfusion $(A)$, severe ischaemia with no reperfusion (B), and penumbra (C). In each probe, the development of glycerol, the LP ratio, and glutamate changes are shown (see text for explanations). 
steady state continuous ${ }^{15} \mathrm{O}$ inhalation technique. ${ }^{1920}$

A complete PET session was performed at baseline after microdialysis probe insertion, after about 1 hour of MCAO, and repeatedly after clip removal. The final PET session was started after a mean of 18 hours (12-24 hours) of reperfusion.

The precise locations of the microdialysis probes were identified by PET at the end of each experiment, by substituting the microdialysis perfusion medium with radioactive fluorodeoxyglucose (FDG) solution. ${ }^{8} \mathrm{~A}$ circular region of interest (ROI), $1 \mathrm{~cm}$ in diameter, was delineated around the identified probe region in the most basal slice showing activity. A corresponding ROI in the contralateral region was delineated so that a side to side ratio between the probe ROI value and the nonaffected side could be calculated for each PET parameter. The ROIs were copied to all PET scans. Microdialysis probes were then classified during MCAO according to the metabolic threshold level of irreversible ischaemia demonstrated previously ${ }^{9}$ as severe ischaemia $\left(\mathrm{CMRO}_{2}<60 \%\right.$ of the corresponding contralateral region) or penumbra $\left(\mathrm{CMRO}_{2}\right.$ decreased but $\geqslant 60 \%$ and OER $>125 \%$ of the corresponding contralateral region).

STATISTICAL METHODS

The Wilcoxon signed rank test for paired observations was used to compare occlusion and late reperfusion values with baseline. Mean values of 2 hour intervals were used in every non-parametric statistical analysis. For baseline data, the 2 hour interval immediately preceding MCAO was used. For the statistical analysis, mean values of the two probes from each monkey were used, except for the probes from one animal, which were allocated to different groups according to the applied PET definitions. Three probes were excluded due to technical problems. The correlations between glycerol and the LP ratio as well as between glycerol and glutamate were tested using Spearman's $r$ test at MCAO and late in reperfusion. $\mathrm{p}$ Values $<0.05$ were considered to be statistically significant.

\section{Results}

Eight probe regions were classified as severe ischaemia on PET; four of these showed characteristic reperfusion when the clip was removed and in the remaining four, no or minimal reperfusion was seen. Five probes were in penumbra regions, all with reperfusion.

In probe regions with severe ischaemia $(n=8)$ there was a nine-fold increase in interstitial glycerol compared with baseline (fig 1). The maximal values were obtained during the first 4 hours after clip removal; after this the glycerol concentration declined to a plateau value around four times baseline, irrespective of the degree of reperfusion. In penumbra probes $(n=5)$, the maximal increase was about fivefold after MCAO, but in these probes the glycerol concentration returned to normal within 8 hours of reperfusion. For all probes, interstitial glycerol was significantly increased during occlusion compared with baseline $(\mathrm{p}=0.012)$.

The slope of glycerol increase was not as steep as that of the LP ratio (fig 2 A and B). Response time (time to doubling of the baseline value) was more than double for glycerol compared with the LP ratio (table 2). Another measure of response time-time to first peak-was about three times as long as that for the LP ratio (table 2). The response time of glutamate was rather variable, resulting in a median value between that of the LP ratio and glycerol in severe ischaemia. In the penumbra, the response time was not calculated because the peaks were small and indistinct.

There was a correlation between glycerol and the LP ratio during MCAO ( $\mathrm{p}=0.028)$ and after 18 hours of reperfusion $(p=0.014)$, reflecting the fact that large increases in the LP ratio were accompanied by parallel high increases in glycerol. Glycerol was also correlated with glutamate at late reperfusion $(p=0.023)$, but not during occlusion $(\mathrm{p}=0.911)$

Graphs of interstitial glycerol, the LP ratio, and glutamate in typical probes located in regions with severe ischaemia with reperfusion, severe ischaemia without significant reperfusion, and penumbra, respectively, are shown in figure 2. Note how in severe ischaemia with reperfusion, glycerol peaked later than both the LP ratio and glutamate, but the increase was more sustained (fig $2 \mathrm{~A}$ ). In the probe with no reperfusion, the glycerol pattern was similar, but the LP ratio and glutamate concentrations displayed secondary increases (fig $2 \mathrm{~B}$ ). In penumbra regions, all three parameters were slightly increased, and returned to baseline within 8 hours after the clip was removed (fig 2C).

\section{Discussion}

About one third of neural tissue cell membrane is phospholipid. The degradation of phospholipids to glycerol is schematically illustrated in figure 3. There is a continuous physiological turnover of phospholipid due partly to the phospholipases acting as effectors in $\mathrm{G}$ protein coupled transmembrane receptor signal processing. Several studies have shown that glycerol concentrations rise during cerebral ischaemia and short periods of reperfusion. ${ }^{21} 22$ With the microdialysis technique, more information about cerebral ischaemia and temporal changes in glycerol concentrations can be obtained. To our knowledge, the present study is the first to investigate the temporal changes of interstitial glycerol in direct relation to regional PET measurements of cerebral blood flow and oxygen metabolism during MCAO and subsequent reperfusion.

We have shown that a $\mathrm{CMRO}_{2}$ level depressed to less than $60 \%$ of contralateral values during transient MCAO seems to be a stable indicator of irreversible ischaemic injury. Conversely, regions with $\mathrm{CMRO}_{2}$ above the threshold of $60 \%$ have a potential for survival. ${ }^{9}$ The relevance of this threshold level was supported in a study showing characteristic 


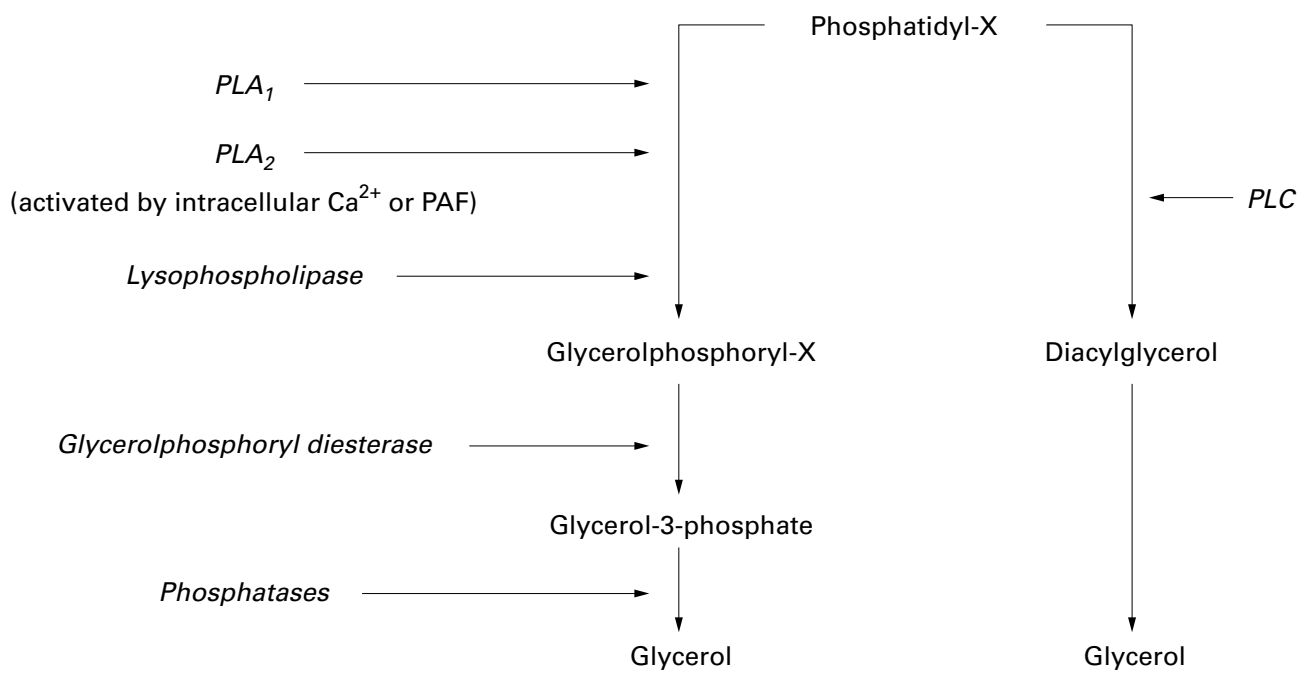

Figure 3 The two parallel biochemical pathways in which membrane phospholipids (phosphatidyl-X) are degraded to glycerol (modified from Gercken and Bräuning ${ }^{2}$ ). Phospholipids are liberated from the cell membrane through the action of phospholipases in normal receptor signal processing. Further degradation to glycerol occurs in the cytosol. In ischaemia, these processes are augmented by for example, $C a^{2+}$ overload. (PL $A_{1}=$ phospholipase $A_{1} ; P L A_{2}=$ phospholipase $A_{2} ; P A F=$ platelet activating factor; $P L C=$ phospholipase $C$ ).

microdialysis profiles for the LP ratio, hypoxanthine, and glutamate in probe regions with $\mathrm{CMRO}_{2}$ below or above the threshold level. ${ }^{8}$ The microdialysis concentrations of energy related metabolites in probe regions classified as severe ischaemia during MCAO were sustained at increased values (suggesting irreversible injury), with secondary increases in probes without reperfusion. By contrast, in penumbra probe regions the microdialysis concentrations normalised during the reperfusion phase. These data support our previous finding that energy related metabolites, particularly the LP ratio, have a high sensitivity and specificity as markers of ischaemia. ${ }^{7}$ In the present study, the glycerol concentration in all severe ischaemia probe regions remained steadily increased after the MCAO phase. Interestingly, this was similar in both severe ischaemia groups, regardless of whether there was significant reperfusion or not. Thus glycerol stands out as a particularly reliable marker of irreversible brain damage, also in comparison with the LP ratio and glutamate. This is in accordance with the traumatic brain injury study by Marklund et al, ${ }^{5}$ in which a sustained interstitial glycerol increase was found in the shear stress zone of a cortical lesion where irreversible neuronal damage occurs.

Energy failure and intracellular $\mathrm{Ca}^{2+}$ overload are major triggering events for membrane phospholipid degradation in cerebral ischaemia. ${ }^{23}$ Some of the $\mathrm{Ca}^{2+}$ influx is caused by an opening of NMDA receptor operated ion channels by glutamate, which accumulates in the interstitial compartment as a result of ischaemia. $^{24}$ These events lead to phospholipase activation and phospholipid degradation (fig 3). This series of events seems to fit well with the present results where severe ischaemia produced an increased LP ratio (mitochondrial dysfunction/energy perturbation), followed by a glutamate increase and eventually raised glycerol concentrations. The persisting LP ratio increase past MCAO suggests an ongoing injury process, irrespective of the degree of reperfusion. This may explain the sustained increase in interstitial glycerol after severe ischaemia.

Membrane damage may also be caused by radical oxygen species (ROS), and brain mitochondria may be an important source of ROS production after cerebral ischaemia. ${ }^{25}$ However, the mitochondria may also be a target for ROS mediated functional impairment. ${ }^{26}$ The role of mitochondrial dysfunction and ROS after cerebral ischaemia was recently reviewed by Fiskum et al..$^{27}$ Although the precise mechanisms are still unclear, recent reports have shown that nitrone ROS scavenger treatment improves energy metabolism and mitochondrial function after $\mathrm{MCAO}$ in rats $^{28}$ and attenuates lactate and glycerol production after traumatic brain injury. ${ }^{29}$ The present results suggest that mitochondrial function is only partly restituted after severe ischaemia, regardless of the degree of reperfusion. Such a functional state favours increased ROS production by the mitochondria. ${ }^{30}$ This may partly explain the sustained glycerol increase during the reperfusion phase.

Does the rise in glycerol solely reflect the breakdown of membrane phospholipid? Another potential source of glycerol could be from the glycolytic pathway, which is outlined in figure 4. In ischaemia, the altered redox state could theoretically divert glycolytic triose phosphate to form glycerol 3-phosphate, oxidizing $\mathrm{NADH}$ to $\mathrm{NAD}^{+}$. An accumulation of glycerol 3-phosphate has been shown to occur in ischaemia in a rat model utilising NMR spectroscopy, which supports the hypothesis that the glycolytic pathway could be a significant source of glycerol in ischaemia. ${ }^{31}$ However, glycerol 3-phosphate is also an intermediate in the breakdown of membrane phospholipid (fig 3). In severe ischaemia, the accumulation of glycerol 3-phosphate may simply reflect membrane damage or phospholipid release. We think that a major contribution 


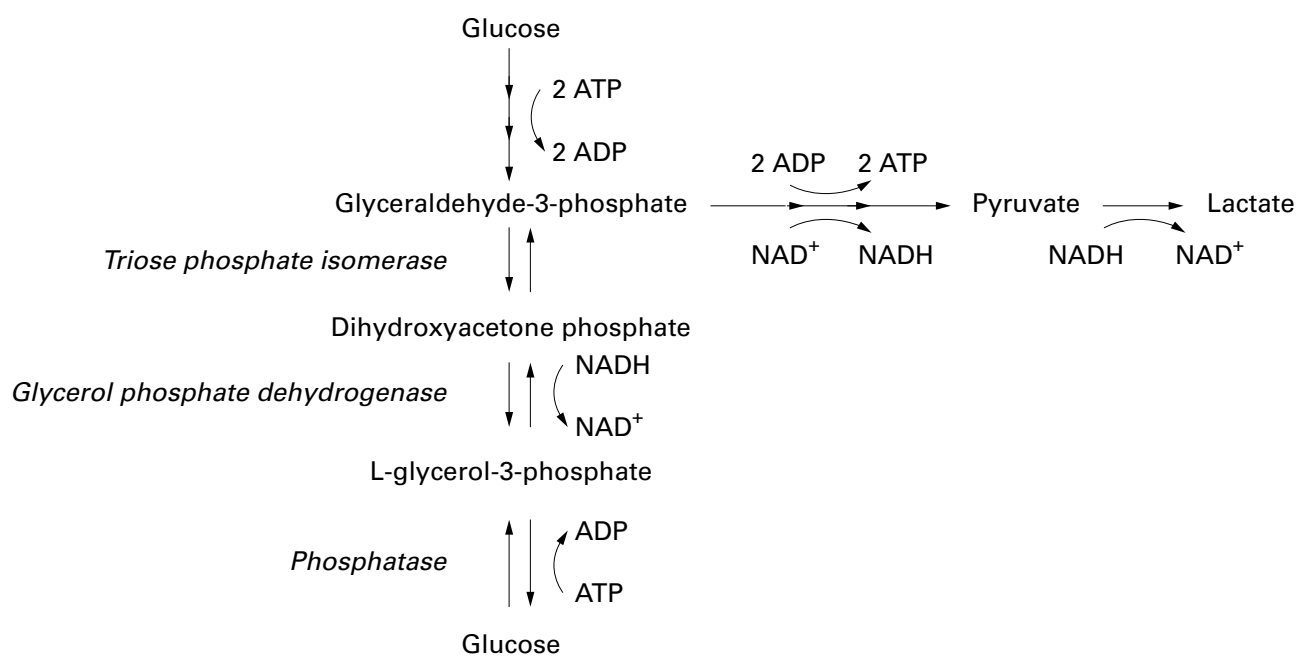

Figure 4 Schematic diagram of the glycolytic pathway. Glycerol can be produced as a byproduct of the glycolytic pathway. Glycerol and glycolytic intermediates are thus interconvertible in normal physiology. In the ischaemic situation, however, shortage of ATP would favour pyruvate and lactate instead of glycerol production.

of glycolysis to glycerol 3-phosphate-and ultimately to glycerol - in this setting is unlikely for two reasons. Firstly, the generation of pyruvate yielding ATP would be favoured, and $\mathrm{NAD}^{+}$is regenerated in the reduction of pyruvate to lactate (fig 4). Should the larger part of the triose phosphate instead end as glycerol, the NADH/ $\mathrm{NAD}^{+}$redox state would be restituted, but there would be a net loss of ATP. Secondly, in severe ischaemia the supply of glucose is stopped, reflected by concentrations approaching zero in microdialysate samples (data not shown). This makes glucose unlikely as a major source of glycerol, especially in severe ischaemia. Another potential source is diffusion of glycerol from the blood across a disrupted blood-brain barrier. Available data suggest that this process does not make a significant contribution to brain interstitial glycerol concentrations in traumatic brain injury and subarachnoid haemorrhage..$^{5}$

Finally, is interstitial glycerol a clinically useful marker of ischaemia? It is obvious that glycerol increase was robust and sustained in severely ischaemic tissue. The concentration was nine times that of baseline after occlusion, and four times baseline during the reperfusion plateau. An interesting difference between the LP ratio and glycerol is that the glycerol shows a sustained increase in all probes in severely ischaemic regions, regardless of the amount of reperfusion. In the context of neuromonitoring, interstitial glycerol could be a reliable marker of irreversible ischaemia, complementary to the LP ratio and glutamate, which are faster to react, but more transient in nature. Perhaps even more important in this context is that interstitial glycerol also seems to be a sensitive marker of reversible ischaemia in the penumbra (a low relative increase with a return to baseline), and could serve as an early warning sign of impending secondary damage.

\section{Conclusions}

Glycerol increased significantly after MCAO. In regions with metabolic signs of irreversible ischaemia, marked, sustained increases were seen. By contrast, the changes in the penumbra were smaller and transient. The study suggests that interstitial glycerol is a sensitive and reliable marker of cell damage in experimental cerebral ischaemia. Further studies are warranted to apply these findings in humans.

We thank Ulla Karlsson and the staff of Uppsala University PET Centre for valuable technical assistance, Lena Nalmo for microdialysis sample analysis, and Professor Gunnar Ronqvist for valuable discussions on glycerol and the glycolytic pathway. The study was supported by grants from the Selander's Foundation, the Swedish Medical Research Foundation, the Mattson Foundation, and the Gävle County Council Research Foundation. The initial phase of the study was supported by "The Subfemtomole Biorecognition Project" under ICORP, Japan Science and Technology Corporation and Uppsala University.

1 Bazán NG. Effects of ischemia and electroconvulsive shock on free fatty acid pool in the brain. Biochim Biophys Acta 1970;218:1-10.

2 Gercken G, Bräuning C. Quantitative determination of hydrolysis products of phospholipids in the ischemic rat brain. Pflugers Arch 1973;344:207-15.

3 Farooqui AA, Horrocks LA. Excitotoxicity and neurological Farooqui AA, Horrocks LA. Excitotoxicity and neurological disorders: involvement of me

4 Chan PH, Longar S, Chen S, et al. The role of arachidonic acid and oxygen radical metabolites in the pathogenesis of vasogenic brain edema and astrocytic swelling. Ann NY Acad Sci 1989;559:237-47

5 Marklund N, Salci K, Lewén A, et al. Glycerol as a marker for post-traumatic membrane phospholipid degradation in rat brain. Neuroreport 1997;8:1457-61.

6 Hillered L, Valtysson J, Enblad P, et al. Interstitial glycerol as a marker for membrane phospholipid degradation in the acutely injured human brain. $\mathcal{F}$ Neurol Neurosurg Psychiatry 1998;64:486-91.

7 Enblad P, Valtysson J, Andersson J, et al. Simultaneous intracerebral microdialysis and positron emission tomography in the detection of ischemia in patients with subarachnoid

8 Enblad P, Frykholm P, Valtysson J, et al. Middle cerebral artery occlusion (MCAO) and reperfusion in primates artery occlusion (MCAO) and reperfusion in primates
monitored by microdialysis and sequential positron emismonitored by microdialysis and sequential

9 Frykholm P, Andersson JL, Valtysson J, et al. A metabolic threshold of irreversible ischemia demonstrated by PET in a middle cerebral artery occlusion-reperfusion primate model. Acta Neurol Scand 2000;102:18-26.

10 Hudgkins WR, Garcia JH. Transorbital approach to the middle cerebral artery of the squirrel monkey: a technique for experimental cerebral infarction applicable to ultrastructural studies. Stroke 1970;1:107-11.

11 O'Brien MD, Waltz AG. Transorbital approach for occluding the middle cerebral artery without craniectomy. Stroke 1973;4:201-6.

12 Hallström Å, Carlsson A, Hillered L, et al. Simultaneous determination of lactate, pyruvate, and ascorbate in microdialysis samples from rat brain, blood, fat, and muscle using high-performance liquid chromatography. Fournal of Pharmacological Methods 1989;22:113-24.

13 Reid MS, Hererra-Marschitz M, Kehr J, et al. Striatal dopamine and glutamate release: effect of intranigral injection of substance P. Acta Physiol Scand 1990;140:527-37. 
14 Foster KJ, Alberti KG, Hinks L, et al. Blood intermediary metabolite and insulin concentrations after an overnight fast: reference ranges for $1978 ; 24: 1568-72$.

15 Benveniste H, Hansen AJ, Ottesen NS. Determination of brain interstitial concentrations by intracerebral microdialysis. F Neurochem 1989;52:729-34.

$16 \mathrm{Kehr}$ J. A survey on quantitative microdialysis: theoretical models and practical implications. I Neurosci Methods 1993;48:251-61

17 Holte S, Eriksson K, Dahlbom M. A preliminary evaluation of the Scanditronix PC2048-15B brain scanner. Eur $\mathcal{F}$ Nuc Med 1989;15:719-21.

18 Andersson JLR. A rapid and accurate method to realign PET scans utilizing image edge information. $7 \mathrm{Nucl} \mathrm{Med}$ 1995;36:657-69.

19 Frackowiak RSJ, Lenzi G-L, Jones T, et al. Quantitative measurement of regional cerebral blood flow and oxygen metabolism in man using ${ }^{15} \mathrm{O}$ and positron emission tomography: theory, procedure and normal values. $7 \mathrm{Com}^{-}$ tomography: theory, procedure and
put Assist Tomogr 1980;4:727-36.

20 Lammertsma AA, Jones T, Frackowiak RSJ, et al. Theoretical study of the steady-state model for measuring regional cerebral blood flow and oxygen utilisation using oxygen-15. f Cereb Blood Flow Metab 1981;5:544-50.

21 Paschen W, van den Kerckhoff W, Hossmann K-A. Glycerol as an indicator of lipid degradation in bicuculline-induced seizures and experimental cerebral ischemia. Metab Brain Dis $1986 ; 1: 37-44$

22 Bertrand N, Ishii H, Spatz M. Regional and temporal glycerol changes induced by forebrain ischemia in gerbils. Neurosci Lett 1992;148:81.
23 Siesjö BK, Katsura K. Ischemic brain damage: focus on lipids and lipid mediators. Adv Exp Med Biol 1992;318:41-56. ids and lipid mediators. Adv Exp Med Biol 1992;318:41-56.
Hillered L, Hallström A, Segersvärd S, et al. Dynamics of Hillered L, Hallström A, Segersvärd S, et al. Dynamics of
extracellular metabolites in the striatum after middle cerebral artery occlusion in the rat monitored by intracerebral microdialysis. F Cereb Blood Flow Metab 1989; 9:607-16.

25 Piantadosi CA, Zhang J. Mitochondrial generation of reactive oxygen species after brain ischemia in the rat. Stroke 1996;27:327-31.

26 Hillered L, Ernster L. Respiratory activity of isolated rat brain mitochondria following in vitro exposure to oxygen radicals. F Cereb Blood Flow Metab 1983;3:207-14.

27 Fiskum G, Murphy AM, Beal MF. Mitochondria in neurodegeneration: acute ischemia and chronic neurodegenerative diseases. F Cereb Blood Flow Metab 1999:19:35169.

28 Kuroda S, Katsura K, Hillered L, et al. Delayed treatment with $\alpha$-phenyl- $n$-tert-butyl nitrone (PBN) attenuates secondary mitochondrial dysfunction after transient focal cerondary mitochondrial dysfunction after transient focal

29 Lewén A, Hillered L. Involvement of reactive oxygen species in membrane phospholipid breakdown and energy perturbation after traumatic brain injury in the rat. $\mathcal{F}$ Neurotrauma 1998;15:521-30.

30 Murphy AN, Fiskum G, Beal MF. Mitochondria in neurodegeneration: bioenergetic function in cell life and death. F Cereb Blood Flow Metab 1999;19:231-45.

31 Ben-Yoseph O, Badar-Goffer RS, Morris PG, et al. Glycerol 3-phosphate and lactate as indicators of the cerebral cytoplasmic redox state in severe and mild hypoxia respectively: 